\title{
Análise de bulas dos medicamentos à base de Ginkgo biloba L. comercializadas em Patos de Minas, Minas Gerais, Brasil
}

\author{
Analysis of the medicine package leaflets containing Ginkgo biloba \\ L. marketed in Patos de Minas, Minas Gerais, Brazil
}

Recebido em: 18/08/2016

Aceito em: 17/04/2017
Érica Belchior de Lima ANDRADE; Lilian Abreu FERREIRA

Curso de Farmácia. Faculdade Patos de Minas. Rua Major Gote, 1408, Centro.

CEP: 38700-001. Patos de Minas, MG, Brasil.E-mail: lyabreu@hotmail.com

\begin{abstract}
Industrialized medicines prepared from the dry extract of Ginkgo biloba L. are found in community pharmacy mainly in the forms of capsules and tablets. In acquiring the industrialized medicine, users have the package insert as the main source of printed information and for this information fulfill their purpose, it must be presented in an objective, updated and complete way. Thus, the objective of the study was to analyze the medicine package inserts containing dry extract of Ginkgo biloba L. according to the standards required by Brazilian normative RDC n. 47/2009 of Brazilian Health Surveillance Agency. An observational study was conducted with analysis of phytomedicines package inserts containing dry extract of Ginkgo biloba $\mathrm{L}$. as single drug in the pharmaceutical form of capsules or tablets, sold in communitarian pharmacies in the city of Patos de Minas in Brazil, considering the topics: Identification of the product, Information for the patient, Legal information and Mandatory statements. Of the total different pharmaceutical specialties found, two did not have their respective package insert. In $45 \%$ of package inserts, some of the Mandatory statements were absent. One package insert brought conflicting information about the actual physical aspect of medicine. About the topic Information to patients, $48 \%$ of the package inserts were incomplete, and among these, with indications not established by law. All package inserts had complete information relating to topics Legal information and Identification of the product. Even under specific legislation, there are still phytomedicines with their package insert out of the established standards. The package inserts may have their primary function diverted when they cease to be an educational and informational material and become a source of confusion and incentive to misuse.
\end{abstract}

Keywords: Herbal Medicine. Medicine Package Inserts. Regulation. Ginkgo biloba.

\section{RESUMO}

Os medicamentos industrializados elaborados a partir do extrato padronizado de Ginkgo biloba L. são encontrados em farmácias comunitárias principalmente nas formas de cápsulas e comprimidos. Ao adquirirem o medicamento industrializado, os usuários têm a bula como principal fonte de informação impressa, e para que as informações contidas nesta cumpram sua finalidade, devem ser apresentadas de maneira objetiva, atualizada e completa. Assim, o objetivo do estudo foi analisar as bulas dos medicamentos à base de extrato seco de Ginkgo biloba L. frente às normas exigidas pela RDC no 47/2009 da Anvisa. Foi realizado um estudo observacional com análise das bulas dos fitoterápicos contendo Ginkgo biloba L. como monofármaco, na forma farmacêutica de cápsulas ou comprimidos, comercializados nas farmácias comunitárias da cidade de Patos de Minas, em relação aos tópicos: Identificação do medicamento, Informações ao paciente, Dizeres legais e Frases obrigatórias. Do total de especialidades farmacêuticas, duas não possuíam bula. Em $45 \%$ das bulas, algumas das frases obrigatórias estavam ausentes. Uma bula trazia informação conflitante sobre o real aspecto físico do medicamento. Das informações aos pacientes, $48 \%$ das bulas apresentaram-se incompletas, algumas com indicações de uso não estabelecidas pela legislação. Todas as bulas apresentaram as informações referentes aos tópicos Dizeres Legais e Identificação do medicamento, completas. Mesmo diante de uma legislação específica, ainda existem fitoterápicos com suas respectivas bulas fora dos padrões estabelecidos. As bulas podem ter sua função precípua desviada quando deixam de ser um material educativo e informativo tornando-se fonte de confusão e incentivo ao uso incorreto.

Palavras-chave: Medicamentos Fitoterápicos. Bulas de Medicamentos. Legislação de Medicamentos. Ginkgo biloba. 


\section{INTRODUÇÃO}

Os fitoterápicos estão entre os medicamentos mais vendidos mundialmente, com uma taxa média de expansão movimentando cerca de 14 bilhões de dólares no mercado mundial. Se compararmos com o mercado dos fármacos sintéticos, os fitoterápicos apresentam vendas de $15 \%$ contra apenas $4 \%$ dos sintéticos, com taxas de crescimento duas vezes mais expressivas (1-6).

Entre os diversos fitoterápicos comercializados no Brasil, o Ginkgo biloba é um dos mais populares. Possui suas ações terapêuticas bem documentadas, sendo indicado em casos de dificuldade de concentração, falhas de memória, depressão, vertigens, zumbidos, dores de cabeça e em dores provenientes de doença arterial periférica oclusiva em pacientes após esforço físico $(3,7,8)$.

Os medicamentos industrializados à base de Ginkgo biloba L. são encontrados no mercado principalmente nas formas de cápsulas e comprimidos preparados a partir do extrato padronizado de Ginkgo biloba L $(2,9)$. Os extratos padronizados devem conter um teor mínimo de 22 a $27 \%$ de ginkgoflavonoides (determinados como quercetina, kaempferol e isoramnetina) e um teor de 5 a 7\% de terpenolactonas (ginkgolídeos A, B, C e J e bilabolídeos) (11).

Ao adquirirem um medicamento industrializado, os pacientes possuem, como principal método informative, a bula; e para que as informações contidas cumpram seu propósito e sejam proficientes, devem ser apresentadas de forma objetiva, atualizada e completa $(4,9,10,12)$.

Antes de ser lançado no mercado, todo e qualquer medicamento, assim como os fitoterápicos, deve ter garantidas qualidade, fórmula padronizada e segurança para a população perante a legislação em vigor. A qualidade deve ser alcançada mediante o controle da matéria-prima do produto acabado, materiais de embalagens, incluindo a bula e todas as informações contidas nela, formulação farmacêutica e estudos de estabilidade $(8,9)$.

A bula é o único material informativo de fácil acesso disponível aos pacientes para informações sobre o medicamento. Como é utilizada por pacientes leigos e sem conhecimentos técnicos, é necessário que ela apresente linguagem clara e contenha todas as informações possíveis. O profissional farmacêutico, responsável pela sua confecção até a dispensação ao consumidor, tem como funções a fiscalização e o controle da rotulagem de embalagens em todas as etapas da cadeia de produção do medicamento $(2,9,10,13)$. As bulas de medicamentos fitoterápicos são regulamentadas pela RDC no 47/2009 da Agencia Nacional de Vigilância Sanitária (Anvisa), e devem ser harmonizadas de acordo com suas respectivas Bulas Padrão para aqueles medicamentos fitoterápicos que as possuem publicadas no site da Anvisa $(10,14)$.

A julgar pela importância da bula para o paciente e para o profissional de saúde, bem como sua fiscalização e atualização pela Anvisa fornecendo ao usuário segurança e eficácia, o objetivo deste estudo foi analisar as bulas de medicamentos à base de Gingko biloba L. na forma de sólidos orais de diferentes fabricantes nacionais, comercializados na cidade de Patos de Minas frente à legislação brasileira exigida para as bulas de medicamentos fitoterápicos.

\section{MATERIAL E MÉTODOS}

Foi realizado um estudo quantitativo do tipo observacional na cidade de Patos de Minas durante os meses de novembro e dezembro de 2015. Foram realizadas visitas às farmácias comunitárias da região central, as quais foram escolhidas aleatoriamente. A cidade foi escolhida pela acessibilidade, e a região central por concentrar o maior número de drogarias da cidade e fluxo de pessoas.

Todas as bulas foram analisadas frente à $\mathrm{RDC} \mathrm{n}^{\circ}$ 47/2009 da Anvisa (10), que regulamenta o texto de bula de medicamentos fitoterápicos e anexa os modelos de bulas padrão das treze espécies vegetais mais comercializadas no Brasil. Foram levados em consideração na análise os seguintes tópicos: informações ao paciente, dizeres legais e frases obrigatórias. Foram observados ainda erros de ortografia e orientação quanto à data de aprovação e atualização disponibilizadas no site da Anvisa.

Foram considerados os seguintes critérios: Ausente (não apresenta o item); Em desacordo (apresenta o item, mas as informações são incorretas); Incompleto (apresenta somente parte da informação exigida) e De acordo (informação apresentada de forma correta).

Os dados foram tabulados e calculados em percentagem no Microsoft Excel, versão 2010. Foi considerado o percentual de $100 \%$ para a amostra dos 10 medicamentos encontrados, sendo incluídos e contabilizados como ausente nos cálculos os medicamentos que não possuíam bulas. 


\section{RESULTADOS E DISCUSSÃO}

Considerando apenas um registro por laboratório, foram encontrados 34 registros de produtos na Anvisa contendo Ginkgo biloba L. como monofármaco na forma farmacêutica de cápsulas ou comprimidos. Destes, somente 10 apresentavam registro com validade ainda vigente.

Foram encontrados, em comercialização, 10 medicamentos contendo o Ginkgo biloba L., na forma farmacêutica de cápsulas ou comprimidos, abrangendo uma amostra de $70 \%$ dos produtos que apresentavam registro na Anvisa com validade ainda vigente.

Dos 10 medicamentos encontrados, dois não possuíam bula e um medicamento não possuía a renovação de registro na Anvisa, indicando assim a comercialização de medicamentos em desacordo com os padrões exigidos pela legislação. A bula é item obrigatório nos medicamentos registrados e comercializados no Brasil e deve ser inclusa dentro da embalagem do medicamento (12). A justificativa da ausência nos medicamentos encontrados foi dada pela $\mathrm{RDC} \mathrm{n}^{\circ} 278$, de 22 de setembro de 2005 da Anvisa (15), entretanto esta resolução dispõe sobre o registro de produtos pertinentes à área de alimentos e foi revogada em 2010.

Em todas as bulas foram encontrados erros de ortografia e tradução e uso de termos em inglês sem necessidade. Os erros mais comuns encontrados foram o uso da palavra "dosagem" ao invés da forma correta dose, e o uso da palavra "overdose", no lugar da forma correta que é superdose ou dose excessiva.

Em um medicamento, a bula trazia informação conflitante quanto ao aspecto físico do medicamento, indicando como aspecto físico das cápsulas as cores verde/ verde, sendo que as cápsulas comercializadas possuíam as cores verde/branco, demonstrando a falta de concordância com a bula.

De acordo com a legislação vigente, as bulas devem conter apenas as informações relativas às apresentações comercializadas dos medicamentos (10).

A RDC no 47/2009 da Anvisa preconiza que seja informada a data de aprovação da Bula Padrão com a qual a bula foi harmonizada e/ou atualizada (10); nestes casos, a Gerência de Medicamentos Específicos, Fitoterápicos, Dinamizados, Notificados e Gases Medicinais (GMESP) e a Coordenação de Bula e Rotulagem de Medicamentos e Produtos Biológicos (CBREM) informam a data que deve ser inserida (14). Diante disso, apenas duas bulas estavam atualizadas de acordo com esse preceito.

$\mathrm{Na}$ análise do primeiro tópico "Identificação do medicamento", todas as bulas estavam em conformidade quanto ao nome comercial, nomenclatura botânica e parte da planta usada. Somente os medicamentos que não possuíam bula foram incluídos na classificação Ausente (Tabela 1).

Tabela 1. Conformidade das bulas de Ginkgo biloba analisadas em relação ao tópico "Identificação do medicamento" (novembro-dezembro de 2015)

\begin{tabular}{|c|c|c|c|c|}
\hline & Ausente & Em desacordo & Incompleto & De acordo \\
\hline \multicolumn{5}{|c|}{ Identificação do medicamento } \\
\hline Nome comercial & $20 \%$ & - & - & $80 \%$ \\
\hline Nomenclatura botânica & $20 \%$ & - & - & $80 \%$ \\
\hline Parte da planta usada & $20 \%$ & - & - & $80 \%$ \\
\hline
\end{tabular}

$\mathrm{Na}$ análise do tópico seguinte "Informação ao paciente" (Tabela 2), merece destaque o item "Onde, como e por quanto tempo posso guardar este medicamento?", que apareceu incompleto em $80 \%$, ou seja, em oito das dez bulas analisadas. Na maioria das bulas era mencionado apenas o cuidado da conservação do produto ao abrigo da luz, calor e umidade. Em algumas estava especificada a temperatura ambiente de armazenamento (entre $15^{\circ} \mathrm{C}$ e $30^{\circ} \mathrm{C}$ ). Em outras, ainda estava acrescentado o cuidado de manter o medicamento em sua embalagem original. Em um estudo realizado em Feira de Santana, Bahia, do total de doze bulas analisadas de G. biloba, quatro não continham o item sobre o armazenamento e quatro estavam incompletas (3).

Também merece destaque o item "Como devo usar este medicamento?", que apareceu incompleto em 70\% das bulas. Neste item, de acordo com a Bula Padrão, devem ser descritas as posologias, incluindo o limite máximo diário de administração expresso em unidades de medida ou unidade farmacotécnica correspondente (14). A dose diária do G. biloba deve estar entre 26,4 e 64,8 mg de ginkgoflavooóides e 6,0 e 16,8mg de terpenolactonas. Todas as bulas apresentavam as doses diárias máximas sob a forma de valores dos marcadores químicos, 
assim como nas bulas analisadas no estudo de Silva e cols (2016) (4). Essa variação dificulta para o paciente saber quanto realmente dessas substâncias está presente no medicamento e o quanto está sendo administrado. É importante ressaltar que o paciente deve ter um bom entendimento sobre a utilização correta do medicamento. Tais informações são indispensáveis, pois poderão interferir no tratamento farmacológico, com consequente não-adesão e insucesso terapêutico.
No item "Quando não devo usar este medicamento?", 60\% das informações estavam incompletas, fato este que pode induzir o usuário a achar que o medicamento não possui toxicidade ou é isento de contraindicações, e ainda que qualquer pessoa independente da idade pode utilizá-lo. Além disso, pode também gerar confusões a respeito de interações medicamentosas por não estar em concordância com a legislação.

Tabela 2. Conformidade das bulas de Ginkgo biloba analisadas em relação ao tópico "Informação ao paciente" (novembro-dezembro de 2015)

\begin{tabular}{l|l|l|l} 
Ausente & Não conforme & Incompleto & De acordo
\end{tabular}

\begin{tabular}{|c|c|c|c|c|}
\hline \multicolumn{5}{|c|}{ Informação ao paciente } \\
\hline Para que este medicamento é indicado? & $20 \%$ & - & $20 \%$ & $60 \%$ \\
\hline Como este medicamento funciona? & $30 \%$ & - & $30 \%$ & $40 \%$ \\
\hline Quando não devo usar este medicamento? & $30 \%$ & - & $60 \%$ & $10 \%$ \\
\hline 0 que devo saber antes de usar este medicamento? & $20 \%$ & - & $10 \%$ & $70 \%$ \\
\hline Onde, como e por quanto tempo posso guardar este medicamento? & $20 \%$ & - & $80 \%$ & - \\
\hline Como devo usar este medicamento? & $20 \%$ & - & $70 \%$ & $10 \%$ \\
\hline 0 que devo fazer quando eu me esquecer de tomar este medicamento? & $20 \%$ & - & - & $80 \%$ \\
\hline Quais os males que este medicamento pode causar? & $20 \%$ & - & $20 \%$ & $60 \%$ \\
\hline 0 que fazer se alguém usar uma quantidade maior que a indicada deste medicamento? & $20 \%$ & - & - & $80 \%$ \\
\hline
\end{tabular}

Ainda no tópico "Informação ao paciente", pode ser destacado o item "Como este medicamento funciona?" que em $30 \%$ as informações estavam ausentes, e em $30 \%$, incompletas, deixando de expressar a frase, "Seu médico é a pessoa mais adequada para lhe dar maiores informações sobre o tratamento, siga sempre suas orientações. Não devem ser utilizadas doses superiores às recomendadas." Do total, $40 \%$ estavam de acordo com a RDC no 47/2009 (10).

No item "Para que este medicamento é indicado?", em $20 \%$ as informações estavam ausentes, deixando os usuários sem a importante informação do objetivo ao qual se destina o medicamento. Em $20 \%$ estas informações estavam incompletas, com indicações não estabelecidas na bula padrão, como zumbidos, tinidos e câimbras. As indicações previstas na bula padrão são "desordens e sintomas decorrentes da deficiência do fluxo sanguíneo cerebral como problemas de memória, função cognitiva, tonturas, dor de cabeça, estágios iniciais de demências (como o Alzheimer e demências mistas) e problemas na retina." (14).

Este fato evidencia a finalidade da indústria farmacêutica em exaltar indicações do medicamento como forma de propaganda e não com as informações em acordo com a resolução (13). Em um estudo realizado por Leite e Branco (2010), 9 das 12 bulas do G. biloba não continham este item, um avanço pôde ser notado em nosso estudo, pois $80 \%$ possuíam a indicação (3).

Em "O que devo saber antes de tomar este medicamento", apenas uma bula trazia a informação de maneira incompleta. A legislação exige que, neste item, seja inserida em negrito, a frase "Este medicamento não deve ser utilizado por mulheres grávidas e em amamentação sem orientação médica ou do cirurgião-dentista.”. Entretanto, a bula continha a frase sem negrito. Além de chamar a atenção, a frase em negrito funciona como um alerta quanto às precauções e advertências, uma medida que favorece o uso racional de medicamentos.

No estudo de Silva e cols (2016), o item "O que devo fazer quando eu me esquecer de usar este medicamento?" não foi encontrado em uma bula das 16 analisadas de Ginkgo (4). Em nosso estudo, em todas as bulas analisadas esse item estava presente. A Bula Padrão do $G$. biloba não contém uma conduta padronizada para esse item, apenas orienta que seja descrita a conduta necessária caso haja esquecimento da administração, porém foram encontradas diversidades entre as bulas analisadas (14). Algumas traziam a orientação ao paciente quanto a não fazer a suplementação da dose esquecida, enquanto outras traziam a orientação para que a dose fosse tomada 
assim que lembrada, desde que não estivesse próxima ao horário da dose seguinte. É importante que haja uma padronização entre as bulas com orientações baseadas em ensaios clínicos bem documentados, para que esta informação não seja uma fonte de confusão.

$\mathrm{O}$ item "Quais os males que este medicamento pode causar?" está relacionado às reações adversas do medicamento que o paciente pode apresentar após a ingestão do medicamento, e em $80 \%$ das bulas analisadas essa informação estava de acordo, o que corrobora com um estudo anterior (4), onde todas as bulas analisadas traziam a informação de forma adequada. Uma melhora significativa foi verificada em comparação ao estudo de Leite e Branco (2010), no qual das 12 bulas analisadas, 8 estavam incompletas e 3 não possuíam o item (3). Em 20\% estas informações não apareceram, pois não continham bula e nem alerta na embalagem do medicamento. A ausência desse tipo de informação coloca o paciente em risco, somado ao fato de que os fitoterápicos são, na maioria das vezes, utilizados sem prescrição médica (16).

Tabela 3. Conformidade das bulas de Ginkgo biloba analisadas em relação ao tópico “Dizeres legais" (novembro-dezembro de 2015)

\section{\begin{tabular}{l|l|l|l} 
Ausente & Em desacordo & Incompleto & De acordo
\end{tabular}}

\begin{tabular}{|c|c|c|c|c|}
\hline \multicolumn{5}{|c|}{ Dizeres legais } \\
\hline Registro no ministério da Saúde & $20 \%$ & - & - & $80 \%$ \\
\hline Responsável técnico & $20 \%$ & - & - & $80 \%$ \\
\hline Nome da empresa & $20 \%$ & - & - & $80 \%$ \\
\hline Endereço da empresa & $20 \%$ & - & - & $80 \%$ \\
\hline Cadastro nacional de pessoa jurídica (CNPJ) & $20 \%$ & - & - & $80 \%$ \\
\hline Telefone de atendimento ao consumidor (SAC) da empresa & $20 \%$ & - & - & $80 \%$ \\
\hline
\end{tabular}

Além disso, casos de hemorragias e queda de pressão associadas ao uso do G. biloba são frequentemente relatados em estudos $(3,13)$.A utilizaçãobenéfica domedicamentopressupõe informações adequadas, as quais se omitidas ou informadas incorretamente podem agravar a condição do paciente.

$\mathrm{Na}$ análise do item "O que fazer se alguém usar uma quantidade maior que a indicada deste medicamento?", no qual é apresentada a informação referente à superdose, $80 \%$ das bulas estavam de acordo, e em $20 \%$, por não conter a bula, essa informação estava ausente, não a tendo também impressa na embalagem. Uma evolução marcante foi verificada em nosso estudo em comparação ao estudo realizado por Leite e Branco (2010), onde 10 das 12 bulas analisadas não continham este item (3).

Em relação ao tópico "Dizeres legais", os percentuais relacionados aos itens do são apresentados na Tabela 3. Este tópico abrange o Responsável Técnico, registro no Ministério da Saúde, o nome e endereço do fabricante e titular do registro, bem como o CNPJ e o telefone do Serviço de Atendimento ao Consumidor (SAC) da indústria. Todas as bulas estavam corretas em relação a esse tópico. No estudo de Leite e Branco (2010) foi encontrado resultado semelhante (3). Somente os medicamentos que não possuíam bula foram incluídos na classificação Ausente.
O tópico "Frases Obrigatórias" abrange as precauções, contraindicações e medidas que favorecem o uso correto dos medicamentos. São alertas quanto ao grau de risco do uso do medicamento na gravidez e no aleitamento. Devem ser escritas em negrito, para chamar a atenção e com fonte que não seja de tamanho inferior à fonte utilizada no restante da bula $(10,14)$. Com a análise realizada no estudo nas bulas dos medicamentos encontrados em comercialização, foi possível notar uma deficiência nessas informações (Tabela 4).

Dentro desse tópico, a frase "Não use medicamento sem o conhecimento do seu médico. Pode ser perigoso à saúde" estava ausente em $40 \%$ das dez bulas analisadas. Esta frase visa o uso racional de medicamentos, e ressalta a importância da avaliação do profissional de saúde a fim de evitar a automedicação. Em $60 \%$ das bulas esta frase estava de acordo com a legislação.

Do ponto de vista toxicológico, deve ser considerado que um medicamento fitoterápico não possui somente efeitos imediatos e facilmente correlacionados à sua ingestão, mas também os efeitos em longo prazo e de forma assintomática, como os carcinogênicos, hepatotóxicos e nefrotóxicos (17).

A frase "Este medicamento não deve ser utilizado por mulheres grávidas e em amamentação sem a orientação médica ou do cirurgião-dentista" estava ausente em $40 \%$ das dez bulas analisadas, e de acordo em $30 \%$ de- 
las. Essa frase estava incompleta em 30\% das bulas, deixando de incluir na frase que o medicamento não pode ser utilizado durante a amamentação.

Em $50 \%$ das bulas, a frase "Este medicamento é contraindicado para menores de 12 anos" estava de acordo, e incompleta em 30\% delas, sem o destaque em negrito exigido pela RDC 47/2009 (10), podendo assim passar despercebida, induzindo ao pensamento de que são medicamentos sem contraindicações em relação a determinada faixa etária.Em $70 \%$ das dez bulas analisadas, as frases "Antes de usar, observe o aspecto do medicamento. Caso ele esteja no prazo de validade e você observe alguma mudança no aspecto, consulte o farmacêutico para saber se poderá utilizá-lo" estavam de acordo, em $20 \%$, ausentes, e em $10 \%$ estas informações apareceram incompletas, constando apenas a observação dos aspectos do medicamento, não incluindo para consultar o farmacêutico, caso seja observada mudança no medicamento ainda dentro do prazo de validade.

A outra frase, "Em caso de uso de grande quantidade deste medicamento, procure rapidamente o socorro médico e leve a embalagem ou bula do medicamento, se possível. Ligue para 0800722 6001, se você precisar de mais orientações", estava de acordo em $70 \%$ das dez bulas, em $20 \%$ esta informação estava ausente, e em $10 \%$ apareceu de forma incompleta, não solicitando para procurar socorro médico e levar a embalagem ou a bula do medicamento em caso de grande ingestão. A frase em destaque continha apenas o número do telefone de atendimento para orientações em casos de intoxicação.

Ainda no tópico "Frases obrigatórias", a frase "Siga a orientação do seu médico, respeitando sempre os horários, as doses e a duração do tratamento" apareceu em $70 \%$ das bulas, estando ausente em $30 \%$ delas. A importância dessa frase está no alerta para o uso e a orientação corretos a fim de assegurar a segurança e eficácia no tratamento. É importante seguir a posologia recomendada para que não haja riscos associados ao uso indiscriminado.

Cada medicamento tem um determinado tempo de ação no organismo, bem como uma concentração ideal a ser mantida no sangue para ser efetivo, ressaltando a importância da inclusão da frase, "Não interrompa o tratamento sem o conhecimento do seu médico", que apareceu em $70 \%$ das bulas, sendo que em $30 \%$ essas informações estavam ausentes.

Em "Informe ao seu médico, cirurgião-dentista ou farmacêutico o aparecimento de reações indesejáveis pelo uso do medicamento. Informe também a empresa através do seu serviço de atendimento", esta frase estava em $70 \%$ das bulas analisadas, e em $30 \%$ estava ausente. Essa informação merece destaque, porque para alguns usuários, existe a falsa concepção de que "medicamento natural, se não fizer bem, mal não faz". Segundo o Sistema de Informações Tóxico-Farmacológicas (SINITOX), os medicamentos ocupam o primeiro lugar entre os agentes causadores de intoxicações em seres humanos (18).

Acrescenta-se ainda a esse tópico, a frase "Informe ao seu médico ou cirurgião-dentista se você esta fazendo uso de algum outro medicamento", que apresentou-se de acordo em $70 \%$ das bulas, e em 30\% estas informações deixaram de aparecer. Esta frase possui grande relevância, devido ao risco de interações medicamentosas com o G. biloba. Na maioria das vezes, um único fármaco não é o suficiente para a recuperação da saúde ou abrange a totalidade de sintomas de um paciente. Desta forma, dois ou mais fármacos poderão ser prescritos e esta combinação nem sempre trará o benefício almejado, devido à interação entre eles. Essa interação não se reduz somente ao universo fármaco-fármaco, pode ocorrer também entre alimentos, chás, bebidas alcóolicas e/ou cafeinados, entre outros.

O Gingko biloba L. pode ter seus efeitos potencializados ou até mesmo anulados quando administrado com outros medicamentos, determinados alimentos e bebidas alcoólicas (19).

A frase "Todo medicamento de ser mantido fora do alcance das crianças" estava de acordo em todas as bulas analisadas. $\mathrm{O}$ armazenamento seguro, em lugares altos e fechados, evitando o alcance das crianças, contribui para a redução dos riscos de intoxicações medicamentosas. Estudos indicam que a população infantil, principalmente os menores de quatro anos de idade, é a mais exposta aos riscos de intoxicações medicamentosas. Esse fato pode ser justificado por características naturais deste grupo etário (como as diferenças farmacodinâmicas e farmacocinéticas), a curiosidade intrínseca a essa fase do desenvolvimento e falta de noção de perigo $(19,20)$. 
Tabela 4. Conformidade das bulas de Ginkgo biloba analisadas em relação ao tópico “Frases obrigatórias" novembro-dezembro de 2015)

\begin{tabular}{l|l|l|l} 
Ausente & Em desacordo & Incompleto & De acordo
\end{tabular}

\begin{tabular}{|c|c|c|c|c|}
\hline \multicolumn{5}{|c|}{ Frases Obrigatórias } \\
\hline Este medicamento é contraindicado para menores de 12 anos. & $20 \%$ & - & $30 \%$ & $50 \%$ \\
\hline $\begin{array}{l}\text { Este medicamento não deve ser utilizado por mulheres grávidas e em } \\
\text { amamentação sem orientação médica ou do cirurgião-dentista. }\end{array}$ & $40 \%$ & - & $30 \%$ & $30 \%$ \\
\hline $\begin{array}{l}\text { Informe ao seu médico ou cirurgião-dentista se você está fazendo uso } \\
\text { de algum outro medicamento. }\end{array}$ & $30 \%$ & - & - & $70 \%$ \\
\hline $\begin{array}{l}\text { Não use medicamento sem o conhecimento do seu médico. Pode ser } \\
\text { perigoso para a sua saúde. }\end{array}$ & $40 \%$ & - & - & $60 \%$ \\
\hline Número de lote e datas de fabricação e validade: vide embalagem. & $20 \%$ & - & - & $80 \%$ \\
\hline $\begin{array}{l}\text { Não use medicamento com o prazo de validade vencido. Guarde-o em } \\
\text { sua embalagem original. }\end{array}$ & $20 \%$ & - & - & $80 \%$ \\
\hline $\begin{array}{l}\text { Antes de usar, observe o aspecto do medicamento. Caso ele esteja no } \\
\text { prazo de validade e você observe alguma mudança no aspecto, consulte } \\
\text { o farmacêutico para saber se poderá utilizá-lo. }\end{array}$ & $20 \%$ & - & $10 \%$ & $70 \%$ \\
\hline Todo medicamento deve ser mantido fora do alcance das crianças. & $20 \%$ & - & - & $80 \%$ \\
\hline $\begin{array}{l}\text { Siga a orientação de seu médico, respeitando sempre os horários, as } \\
\text { doses e a duração do tratamento. }\end{array}$ & $30 \%$ & - & - & $70 \%$ \\
\hline Não interrompa o tratamento sem o conhecimento do seu médico. & $30 \%$ & - & - & $70 \%$ \\
\hline $\begin{array}{l}\text { Em caso de dúvidas, procure orientação do farmacêutico ou de seu } \\
\text { médico, ou cirurgião-dentista. }\end{array}$ & $30 \%$ & - & - & $70 \%$ \\
\hline $\begin{array}{l}\text { Informe ao seu médico, cirurgião-dentista ou farmacêutico o apare- } \\
\text { cimento de reações indesejáveis pelo uso do medicamento. Informe } \\
\text { também à empresa através do seu serviço de atendimento. }\end{array}$ & $30 \%$ & - & - & $70 \%$ \\
\hline $\begin{array}{l}\text { Em caso de uso de grande quantidade deste medicamento, procure } \\
\text { rapidamente socorro médico e leve a embalagem ou bula do medica- } \\
\text { mento, se possível. Ligue para } 08007226001 \text {, se você precisar de mais } \\
\text { orientações. }\end{array}$ & $20 \%$ & - & $10 \%$ & $70 \%$ \\
\hline
\end{tabular}

A frase "Número de lote e datas de fabricação e validade: vide embalagem" aparece de acordo em todas as bulas analisadas. Apesar dos dois medicamentos encontrados não possuírem bula, essa informação estava presente na embalagem.

Em $80 \%$ das bulas, a frase "Não use medicamento como prazo de validade vencido. Guarde-o em sua embalagem original" estava de acordo com a norma exigida, e em $20 \%$ dessas bulas esta informação estava ausente.

Os pacientes devem entender que os medicamentos devem ser armazenados em sua embalagem original, para que possam ser facilmente identificados e seu prazo de validade monitorado, não havendo assim, possibilidade de troca $(19,20)$.

E por fim, a frase "Em caso de dúvidas, procure orientação do farmacêutico ou do seu médico, ou cirurgião-dentista" apareceu de acordo em $70 \%$ das dez bulas analisadas, e em $30 \%$ dessas bulas esta informação estava ausente. É imprescindível que o usuário seja alertado a não seguir indicações de pessoas não habilitadas. Os únicos capacitados a sanar dúvidas sobre medicamentos são os profissionais em destaque na frase.
De modo geral, foi verificado que existem ainda inúmeras inadequações relacionadas às bulas de medicamentos a base de G. biloba.A ausência de informação ou a presença de informação incompleta foi claramente observada na maioria das bulas analisadas, principalmente em relação ao tópico "Informações ao paciente".

Mesmo após quase oito anos da data de publicação da resolução que regulamenta as bulas de fitoterápicos no Brasil e em comparação a estudos anteriores realizados em outras cidades $(3,4,13,21)$, ainda há medicamentos sendo comercializados em desacordo aos padrões da legislação vigente. No estudo de Bello e cols (2010), realizado em Porto Alegre no ano de 2001, somente 51\% dos fitoterápicos estudados possuíam bula (3). Uma evolução foi notada no estudo de Maia e cols (2012), onde $100 \%$ dos fitoterápicos apresentavam bula (21). Considerando que nesse estudo ainda foram encontrados em comercialização dois medicamentos sem bula, não se pode deixar de pontuar que é imprescindível que os órgãos competentes intensifiquem a fiscalização, para que se cumpram as orientações da Anvisa. 


\section{CONCLUSÃO}

Foi possível observar a falta de concordância e padronização nas informações das bulas analisadas, e que ainda existem muitos medicamentos fitoterápicos sendo comercializados na cidade de Patos de Minas que não atendem à normatização estabelecida pela Anvisa. A bula é a principal fonte de informação escrita para os pacientes que fazem uso de um medicamento. Portanto, quando informações não estão presentes ou aparecem de forma incompleta, podem acarretar prejuízos quanto a um tratamento eficaz, uma vez que o paciente pode não compreender seu conteúdo. Assim, as bulas podem ter sua função precípua desviada quando deixam de ser um material educativo e informativo para se tornar uma fonte de confusão e incentivo ao uso incorreto.

Os dados obtidos mostraram a necessidade de fiscalização das bulas pelos órgãos competentes para que os usuários de medicamentos fitoterápicos a base de Gingko biloba L. possam ter acesso a informações corretas, mais claras, seguras e eficientes para o uso destes medicamentos e para que assim a bula cumpra sua fundamental importância quanto à promoção ao uso racional do medicamento fitoterápico.

\section{REFERÊNCIAS}

1. Pinto JM, Silveira JG. Bulas de medicamentos comercializados no Brasil: em foco a análise da qualidade da informação. Tend Pesq Bras Ciência Inform. 2014; 7:122.

2. Korolkovas A, França FFAC. Dicionário terapêutico Guanabara. 21. ed. Rio de Janeiro: Guanabara Koogan; 2014.

3. Leite TCC, Branco A. Análises das bulas de medicamentos à base de Gingko biloba L. Rev Ciên Farm Básica Apl. 2010; 31(1): 83-87.

4. Silva J, Passos M, Simas N, Santos M. Qualidade das bulas de fitoterápicos no Brasil. Vigil san debate. 2016; 4(2): 56-64. DOI: 10.3395/2317-269x.00677.

5. Carvalho ACB, Perfeito JPS, Costa e Silva LV, Ramalho LS, Marques RFO, Silveira D. Regulation of herbal medicines in Brazil: advances and perspectives. Braz J Pharmac Sci. 2011; 47(3): 467-473. DOI: 10.1590/ S1984-82502011000300004.

6. Rodrigues W. Competitividade e mudança institucional na cadeia produtiva de plantas medicinais no Brasil. Interações (Campo Grande). 2016; 17(2): 267-277. DOI: $10.20435 / 1984042$ X2016210.

7. Silva, Daniella T. Análise comparativa de embalagens contendo Ginkgo biloba L. [TCC - Especialização]. Rio de Janeiro: Instituto de Tecnologia em Fármacos/Farmanguinhos, Fundação Oswaldo Cruz; 2013.

8. Diamond BJ, Bailey MR. Ginkgo biloba: Indications, mechanisms, and safety. Psychiatr Clin North Am. 2013; 36(1):73-83. DOI: 10.1016/j.psc.2012.12.006.

9. Fujita PL, Mac CJS, Teixeira MO. A bula de medicamentos e a regulação de suas configurações em termos de forma e conteúdo no Brasil. Saúde Soc. 2014; 23(1): 277-292. DOI: 10.1590/S0104-12902014000100022.

10. BRASIL. Ministério da Saúde. Agência Nacional de Vigilância Sanitária (Anvisa). Resolução da Diretoria
Colegiada $n^{\circ} 47$ de 08 de setembro de 2009. Estabelece regras para elaboração, harmonização, atualização, publicação e disponibilização de bulas de medicamentos para pacientes e para profissionais de saúde. [acesso em 10 ago 2015] Disponível em: http://www.anvisa.gov.br/ medicamentos/bulas/rdc_47.pdf.

11. WHO -World Health Organization. Folium Ginkgo. In: World Health Organization. Monographs on selected medicinal plants. Geneva: World Health Organization; 2009. [acesso em fev 2016] Disponível em: http://apps. who.int/medicinedocs/en/d/Js2200e/.

12. Caldeira TR, Neves ERZ, Perini E. Evolução histórica das bulas de medicamentos no Brasil. Cad Saude Pub. 2008; 24(4):737-743. DOI: 10.1590/S0102311X2008000400003.

13. Bello CM, Montanha JA, Shenkel EP. Análise de bulas de medicamentos fitoterápicos comercializados em Porto Alegre, RS, Brasil. Rev Bras Farmacogn. 2002; 12(2): 75-83. DOI: 10.1590/S0102-695X2002000200004.

14. BRASIL. Ministério da Saúde. Agência Nacional de Vigilância Sanitária (ANVISA). Bulas padrão de medicamentos fitoterápicos. [acesso em 23 mar 2016] Disponível em: http://portal.anvisa.gov.br/bulas-padrao-de-medicamentos-fitoterapicos.

15. BRASIL. Ministério da Saúde. Agência Nacional de Vigilância Sanitária (Anvisa). Resolução da Diretoria Colegiada $n^{\circ} 278$, de 22 de setembro de 2005. Aprova as categorias de Alimentos e Embalagens Dispensados e com Obrigatoriedade de Registro. [acesso em 23 mar 2016] Disponível em: http://bvsms.saude.gov.br/bvs/saudelegis/anvisa/2005/res0278_22_09_2005.html.

16. Santana IC, Ferreira RC, Peruchetti D, Bachinski N, Scaramello CBV. Perfil de utilização de fitoterápicos por estudantes universitários da Universidade do Grande Rio (UNIGRANRIO). Rev Bras Farm. 2008; 89(4): 311-314. 


\section{Inoarina}

17. Nicoletti MA, Oliveira-Junior MA, Bertasso CC, Caporossi PY, Tavares APL. Principais interações no uso de medicamentos fitoterápicos. Infarma. 2007; 19(1):32-40.

18. BRASIL. Fundação Instituto Oswaldo Cruz. Centro de Informação Científica e Tecnológica. Sistema Nacional de Informações Tóxico - Farmacológicas -SINITOX. Plantas tóxicas no Brasil, 2009. [acesso em: 23 mar. 2016] Disponível em: http://sinitox.icict.fiocruz.br/dados-nacionais.

19. Maior MCLS, Oliveira VBV. Intoxicação medicamentosa infantil: um estudo das causas e ações preventivas possíveis. Rev Bras Farm. 2012; (93):422-430.
20. Nicoletti MA, Itoll RK, Fukushimall AR, Leandro AC. Farmacovigilância de drogas vegetais e seus derivados: uma ação necessária e já iniciada para a segurança do paciente, no contexto do uso racional de medicamentos. Vigil Sanit Debate. 2015; 3(2):136-143. DOI: 10.3395/2317-269x.00254.

21. Maia JQ, Calou IBF, Gonçalves DO, Bezerra SB, Olinda TM. Análise de bulas de medicamentos fitoterápicos comercializados em municípios do interior do Ceará, Brasil. Rev Bras Farm. 2012; 93(1): 22-26. 